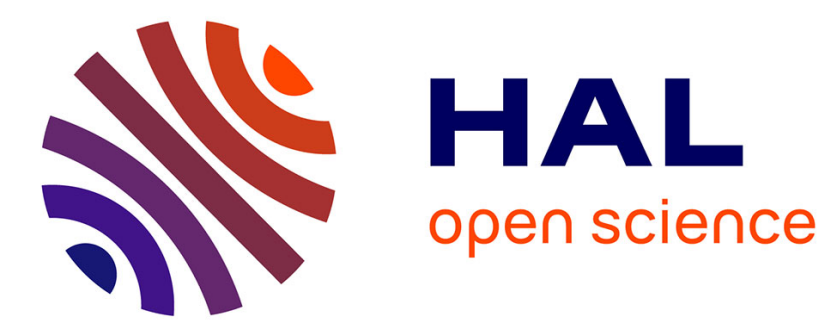

\title{
Blind frame synchronization for error correcting codes having a sparse parity check matrix
}

\author{
Rodrigue Imad, Guillaume Sicot, Sébastien Houcke
}

\section{To cite this version:}

Rodrigue Imad, Guillaume Sicot, Sébastien Houcke. Blind frame synchronization for error correcting codes having a sparse parity check matrix. IEEE Transactions on Communications, 2009, 57 (6), pp.1574-1577. 10.1109/TCOMM.2009.06.070445 . hal-02117781

\section{HAL Id: hal-02117781 \\ https://hal.science/hal-02117781}

Submitted on 2 May 2019

HAL is a multi-disciplinary open access archive for the deposit and dissemination of scientific research documents, whether they are published or not. The documents may come from teaching and research institutions in France or abroad, or from public or private research centers.
L'archive ouverte pluridisciplinaire HAL, est destinée au dépôt et à la diffusion de documents scientifiques de niveau recherche, publiés ou non, émanant des établissements d'enseignement et de recherche français ou étrangers, des laboratoires publics ou privés. 


\title{
Blind frame synchronization for error correcting codes having a sparse parity check matrix
}

\author{
Rodrigue Imad, Guillaume Sicot and Sebastien Houcke
}

\begin{abstract}
We present in this letter a blind frame synchronization method based on a Maximum A Posteriori probability (MAP) approach. Applied to coded communication systems, this method is based on the calculation of the Log-likelihood Ratios (LLR) of the syndrome obtained according to the parity check matrix of the code. After presenting the proposed method, we compare it to an existing blind synchronization method previously introduced. Simulation results show that the proposed method outperforms the existing one and presents good performance when applied to codes having a sparse parity check matrix such as Low Density Parity Check (LDPC) codes and convolutional codes.
\end{abstract}

Index Terms-Blind frame synchronization, channel coding, MAP, LDPC codes, convolutional codes.

\section{INTRODUCTION}

C HANNEL coding is considered to be an important step in digital communication systems. At the reception and before decoding the received symbols, one should be able to find the beginning of each codeword so that the decoder functions properly. This step is called Frame Synchronization. Conventional frame synchronization is achieved by adding to the transmitted symbols a synchronization sequence known by the receiver. This sequence is detected at the reception by running the received symbols through a correlator [1], [2], [3]. Actual codes are very powerful and are able to decode heavily corrupted sequences. In order to maintain good performance in such cases, conventional synchronization methods should increase the length of the inserted synchronization word, which reduces the spectral efficiency of the system. Another solution to improve the system performance is to take advantage of the code structure and consider frame synchronization jointly with the decoding as in [4], [5]. Rather than placing the synchronization bits in a separate header, the authors of [6] propose to place them in a midamble. In [7], a unified approach to frame synchronization has been described where the observed symbols are modeled as the output of a Markov chain corrupted by additive white Gaussian noise.

In this letter, we present a blind method of frame synchronization wherein no additional sequence is added to the coded one. This synchronization method is based on a MAP approach in the sense of minimizing the probability of false frame synchronization of a coded system, given the received signal. It has been first introduced for a Binary Symmetric Channel (BSC) [8] and then generalized for a Gaussian Channel [9].

R. Imad, G. Sicot and S. Houcke are with the Institut TELECOM; TELECOM Bretagne; UMR CNRS 3192 Lab-STICC. Technopôle Brest Iroise, CS 83818, 29238 Brest Cedex 3. Université Européenne de Bretagne, France email: <firstname $>$.<lastname $>@$ telecom-bretagne.eu
This method has been also applied in an Interleaved Division Multiple Access (IDMA) context [10] and it gave promising results. In the literature, we found only one method of blind frame synchronization introduced by Sun and Valenti [11], [12]. The authors claim to have developed an optimal MAP frame synchronizer. However, they have missed an important detail in the expression of the a posteriori probability, which leads to an erroneous MAP estimator. In the remaining of this letter, Sun and Valenti's method will be referred to as the "existing" one and our method as the "proposed" one.

This letter is organized as follows. In Section the proposed synchronization method and briefly present the existing one. Simulation results are shown in Section [II] where the two synchronization methods are applied to LDPC and convolutional codes. Finally, Section [V concludes the work.

\section{PROPOSED METHOD OF BLIND FRAME SYNCHRONIZATION}

In this letter, we consider that the transmitter is sending a binary sequence of codewords and is using a Binary Phase Shift Keying (BPSK) modulation. The propagation channel is corrupted by a quasi-static additive white Gaussian noise. For simplicity reasons, we assume that perfect bit synchronization is achieved. Indeed, our proposed synchronization method works well even on low signal to noise ratios and therefore, it is able to deal with small errors in the timing recovery since such errors just increase the bit error rate of the system.

For a given code of rate $\rho=\frac{n_{c}-n_{r}}{n_{c}}$, it is often possible to find the corresponding parity check matrix $H$ of size $n_{r} \times n_{c}$, where $n_{c}$ represents the length of a codeword and $n_{r}$ the number of parity relations. Let $b(i)= \pm 1$ be the $i^{\text {th }}$ coded and modulated bit to be transmitted. At the reception, the $i^{\text {th }}$ received sample is given by $r(i)=b\left(i-t_{0}\right)+w(i)$, where $t_{0}$ is an integer representing the shift of the transmitted symbols due to the delay introduced by the propagation channel and $w(i)$ is a white Gaussian noise. The received sequence of $N$ samples can then be written as $r=[r(1), \ldots, r(N)]$.

The main target of frame synchronization is to find the position of a codeword in the received sequence. In other words, we have to estimate the delay $t_{0}$ that we assume (without loss of generality) to be lower than $n_{c}$. At a position $t$ on the received sequence $\left(t \in\left[0, n_{c}-1\right]\right)$, we apply a sliding window of length $K n_{c}$ samples, where $K$ is an integer greater or equal to one. The study of all the blocks contained in the $n_{c}$ possible positions of this sliding window allows us to determine whether a position corresponds to the correct synchronization moment or not. In the remaining of this letter 
we assume that $K=1$. This means that only one block is contained in the sliding window.

Our proposed method is based on a MAP approach in the sense of maximizing the probability that a position $t$ corresponds to the correct synchronization moment, given the received samples. In other words, it maximizes the following a posteriori probability:

$$
\operatorname{Pr}[t / r], t \in\left[0, n_{c}-1\right] .
$$

In order to confirm that a position $t$ is the correct synchronization position, we should verify that the block transmitted at this position is a valid codeword and that all the other blocks in the remaining $\left(n_{c}-1\right)$ tested positions are not. The easiest way to check whether a block corresponds to a valid codeword or not is by calculating its syndrome which is obtained according to the parity check matrix $H$ of the code. Each element of the syndrome is calculated using one parity check equation defined by one row of $H$. At a position $t$ of the sliding window, the syndrome can be written as $S_{t}=\left[S_{t}(1), S_{t}(2), \ldots, S_{t}\left(n_{r}\right)\right]$. At the synchronized position, the probability of having a verified parity check equation is greater than the one at a nonsynchronized position. This is due to the fact that at the synchronized position, the sliding window contains a block corresponding to a valid codeword. In the logarithmic domain, these probabilities can be expressed in Log-Likelihood Ratios (LLR).

Let $\psi(t)$ be the LLR of the syndrome calculated on position $t$ of the sliding window.

$\psi(t)=\log \left(\frac{\operatorname{Pr}\left[\left[S_{t}(1), \ldots, S_{t}\left(n_{r}\right)\right] \neq 0\right]}{\operatorname{Pr}\left[\left[S_{t}(1), \ldots, S_{t}\left(n_{r}\right)\right]=0\right]}\right)=\log \left(\frac{1-P_{t}}{P_{t}}\right)$.

For codes having a sparse parity check matrix, the syndrome elements can be assumed independent and therefore:

$$
P_{t}=\operatorname{Pr}\left[\left[S_{t}(1), \ldots, S_{t}\left(n_{r}\right)\right]=0\right]=\prod_{k=1}^{n_{r}} \operatorname{Pr}\left[S_{t}(k)=0\right] .
$$

Let $L\left(S_{t}(k)\right)=\log \left(\frac{\operatorname{Pr}\left[S_{t}(k)=1\right]}{\operatorname{Pr}\left[S_{t}(k)=0\right]}\right)$ be the LLR of the $k^{t h}$ syndrome element,

$$
\operatorname{Pr}\left[S_{t}(k)=0\right]=\frac{1}{1+\exp \left(L\left(S_{t}(k)\right)\right.} .
$$

Using (2), (3), (4) and having $\prod_{k=1}^{n_{r}}\left(1+\exp \left(L\left(S_{t}(k)\right)\right)\right)$ much larger than 1 , the LLR of the syndrome can be written as:

$$
\psi(t)=\sum_{k=1}^{n_{r}} \log \left(1+\exp \left(L\left(S_{t}(k)\right)\right)\right)
$$

and the frame synchronization position is estimated by the one that minimizes the LLR of the syndrome:

$$
\hat{t}_{0}=\underset{t=0, \ldots, n_{c}-1}{\operatorname{argmin}}\{\psi(t)\} .
$$

For simplification reasons, 5 can be approximated by:

$$
\phi(t)=\sum_{k=1}^{n_{r}} L\left(S_{t}(k)\right)
$$

Equation (7) is equivalent to define the LLR of the syndrome by:

$$
\phi(t)=\log \left(\frac{\operatorname{Pr}\left[\left[S_{t}(1)=1, \ldots, S_{t}\left(n_{r}\right)=1\right]\right]}{\operatorname{Pr}\left[\left[S_{t}(1), \ldots, S_{t}\left(n_{r}\right)\right]=0\right]}\right),
$$

which can be interpreted as the worst case scenario: either all parity check equations are verified or none.

Finally, the proposed blind frame synchronizer estimates the frame synchronization position by:

$$
\hat{t}_{0}=\underset{t=0, \ldots, n_{c}-1}{\operatorname{argmin}}\{\phi(t)\} .
$$

Note that the LLR of a syndrome element is given by:

$$
L\left(S_{t}(k)\right)=(-1)^{u_{k}+1} \operatorname{atanh}\left(\prod_{j=1}^{u_{k}} \tanh \left(\frac{\tilde{r}\left(t+k_{j}\right)}{2}\right)\right),
$$

where $\tilde{r}(i)=\frac{2}{\sigma^{2}} r(i)$ is the LLR of the $i^{t h}$ received sample and $\sigma^{2}$ is the variance of the noise. $u_{k}$ and $k_{j}$ represent the number of ones in the $k^{t h}$ row of the parity check matrix of the code and the position of the $j^{\text {th }}$ non zero element in this $k^{\text {th }}$ row, respectively.

According to [13], an approximation of (10) is given by:

$L\left(S_{t}(k)\right)=(-1)^{u_{k}+1}\left(\prod_{j=1}^{u_{k}} \operatorname{sign}\left(\tilde{r}\left(t+k_{j}\right)\right)\right) \min _{j=1, \ldots, u_{k}}\left|\tilde{r}\left(t+k_{j}\right)\right|$.

Having $\tilde{r}(i)$ and $r(i)$ proportional and by assuming the variance of the noise constant during a transmission, $\tilde{r}\left(t+k_{j}\right)$ can be replaced by $r\left(t+k_{j}\right)$ in 11. In this way, no $a$ priori information about the AWGN channel is required [14]. $L\left(S_{t}(k)\right)$ and $\phi(t)$ are then proportional to:

$$
\begin{aligned}
& \hat{L}\left(S_{t}(k)\right)=(-1)^{u_{k}+1}\left(\prod_{j=1}^{u_{k}} \operatorname{sign}\left(r\left(t+k_{j}\right)\right)\right) \\
& \cdot \min _{j=1, \ldots, u_{k}}\left|r\left(t+k_{j}\right)\right| \\
& \text { and } \hat{\phi}(t)=\sum_{k=1}^{n_{r}} \hat{L}\left(S_{t}(k)\right),
\end{aligned}
$$

and the frame synchronization position is estimated by:

$$
\hat{t}_{0}=\underset{t=0, \ldots, n_{c}-1}{\operatorname{argmin}}\{\hat{\phi}(t)\} .
$$

Comparison with an existing method of blind frame synchronization

Applied in our context, the existing method introduced in [11], [12] aims to maximize the log-likelihood function: $L(t)=-\frac{\left(\phi(t)-n_{r} M_{t_{0}}\right)^{2}}{2 \kappa n_{r} M_{t_{0}}}$, where $n_{r} M_{t_{0}}$ is the mean of $\phi\left(t_{0}\right)$ and $\kappa$ is a coefficient introduced to describe the dependence of the syndrome elements. The concept of this method is very close to ours but with one main drawback. In order to estimate the synchronization position, the authors examine only whether $\phi$ at a position $t$ follows the same law as the one at the synchronized position. However, they do not verify that the remaining $\phi(i)_{i \neq t}$ corresponding to the other $\left(n_{c}-1\right)$ tested positions follow the law of $\phi$ at a nonsynchronized position. In other words, comparing the variables $\phi$ to the mean 


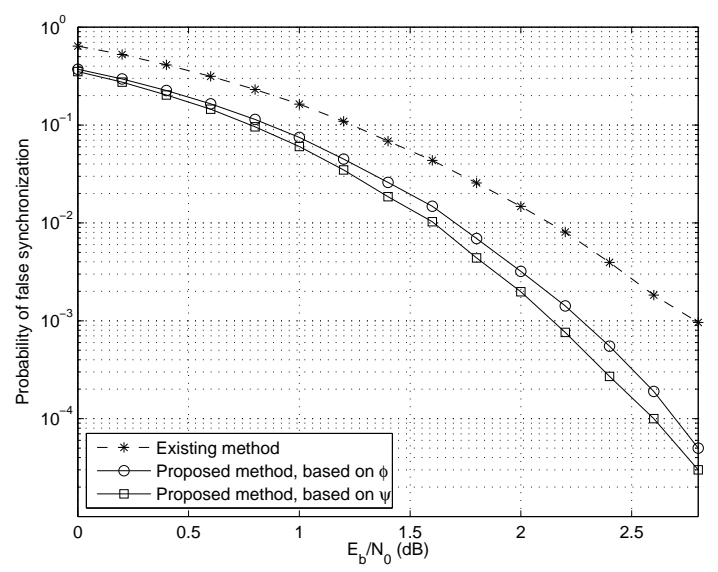

Fig. 1. Comparison between the proposed and existing methods applied to an LDPC code of rate $R=1 / 2$ and length $n_{c}=512$ bits.

of $\phi\left(t_{0}\right)$ is not enough to guarantee an optimum estimation of $t_{0}$. At a nonsynchronized position, we might have a value of $\phi$ closer to the mean of $\phi\left(t_{0}\right)$ than a correct value corresponding to the synchronized one. Hence, it is obvious that the existing method does not afford an optimum estimation of the correct synchronization position.

\section{Simulation RESULtS}

In order to study the performance of our synchronization method, we estimated the probability of false synchronization. The evaluation of this probability is realized by Monte Carlo simulation. For each configuration, the noise, information bits and the delay of the channel were randomly chosen. Fig. 1 shows a comparison between the proposed synchronization method and the existing one once applied to an LDPC code of length 512 bits, having 5 non zero elements in each row of its parity check matrix. First of all, we can see that using (5) or (7) to estimate the frame synchronization position gives almost the same results. Therefore, in the remaining of this letter and for complexity reasons, our proposed method will be based on (7). From Fig. 1, it is clear that the proposed method outperforms the existing one. A gain of around $0.5 \mathrm{~dB}$ is achieved at a probability of false synchronization equal to $10^{-3}$. Fig. 2] shows the same comparison when applying these methods to convolutional codes. Two systematic and recursive convolutional codes of generator polynomials $(1,5 / 7)$ and $(1,23 / 35)$ were tested. These two codes have the same length (512 bits), the same rate $(1 / 2)$ and their constraint lengths equal to 3 and 5 , respectively. Note that the number of non zero elements in the parity check matrix increases with the constraint length of the code. Consequently, a degradation in performance is observed [9]. Compared to the existing method, our proposed blind frame synchronization method yields always better results.

We also compared our results to the ones obtained with Massey's synchronization approach, which locates a synchronization word periodically embedded in the binary data [2]. This comparison is done in Fig. 3 for the convolutional code $(1,5 / 7)$. Three different lengths of a synchronization word

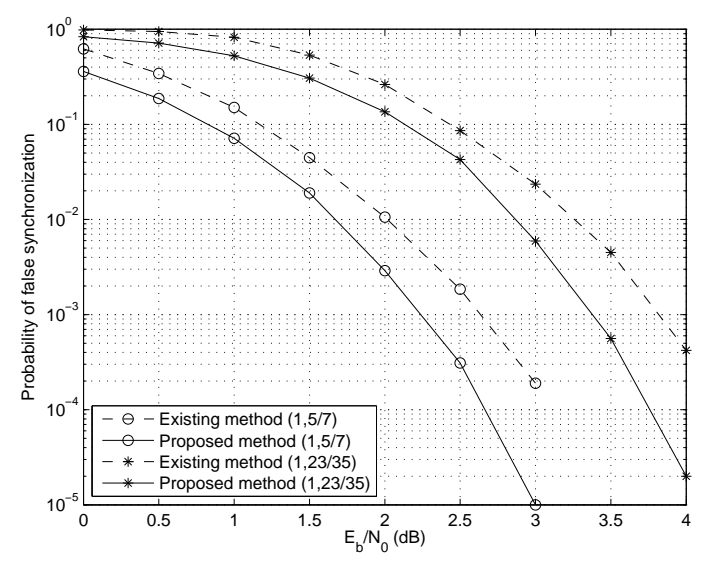

Fig. 2. Comparison between the proposed and existing methods applied to convolutional codes of rate $R=1 / 2$ and length $n_{c}=512$ bits.

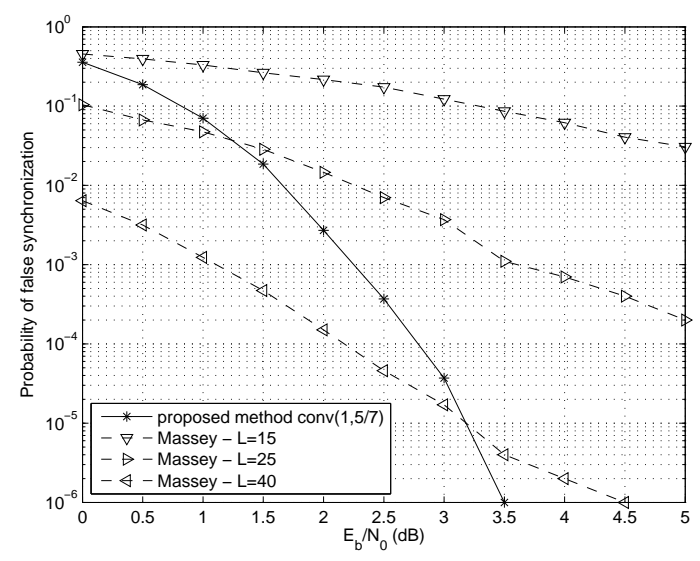

Fig. 3. Comparison between Massey's method and the proposed one.

were tested, $L=15,25$ and 40 bits. We can clearly see that our method converges quicker than the one introduced by Massey. Even for a synchronization word of length $L=40$ bits (around $8 \%$ of the total length of the code), our method presents better performance in the zone were the convolutional code is generally used $\left(E_{b} / N_{0}>3.5 \mathrm{~dB}\right)$.

In order to evaluate the robustness of the proposed synchronization method, we plotted in Fig. 4 the Frame Error Rate (FER) curves obtained by decoding the previous convolutional codes using a MAP decoder, which is applied after achieving the blind frame synchronization step. As we can see, the FER curves for code $(1,5 / 7)$ obtained after applying the proposed synchronization method and the ones obtained in the perfect synchronization case are almost the same. A small degradation in performance is observed for code $(1,23 / 35)$. However, for $E_{b} / N_{0}>3.5 d B$, the two curves of FER become one and no degradation in performance is detected.

\section{CONCLUSION}

In this letter, we have proposed an optimum blind frame synchronization method based on a MAP approach. Applied to codes having a sparse parity check matrix such as LDPC codes, the proposed synchronization method presents good 


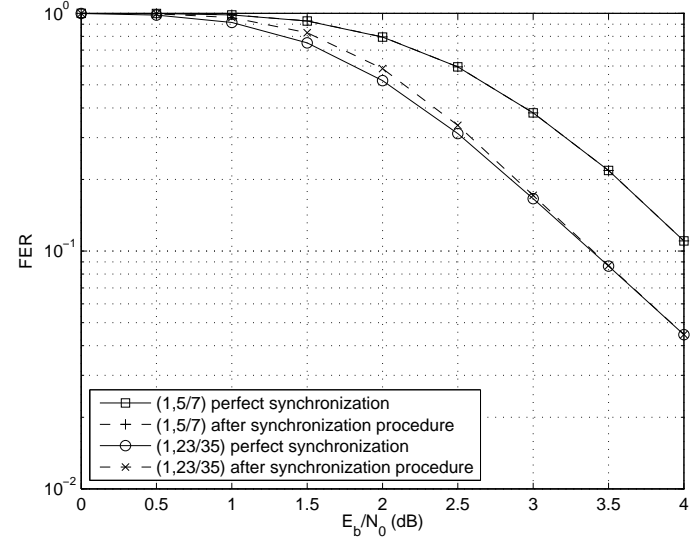

Fig. 4. Frame error rate of convolutional codes with code rate $R=1 / 2$ and length $n_{c}=512$ bits.

performance and clearly outperforms an existing blind frame synchronization method. Furthermore, once the proposed synchronization method is applied to convolutional codes, the frame error rates of the system are almost the same as the ones obtained in the perfect synchronization case.

\section{REFERENCES}

[1] R. H. Barker, "Group synchronization of binary digital systems," Communication Theory, pp. 273-287, 1953.
[2] J. L. Massey, "Optimum frame synchronization," IEEE Transactions on Communications, vol. COM-20, pp. 115-119, April 1972.

[3] G. L. Lui and H. H. Tan, "Frame synchronization for gaussian channels," IEEE Transactions on Communications, vol. COM-35, pp. 818-829, August 1987.

[4] P. Robertson, "A generalized frame synchronizer," in Proc. of GLOBECOM, December 1992, pp. 365-369.

[5] T. M. Cassaro and C. N. Georghiades, "Frame synchronization for coded systems over AWGN channels," IEEE Transactions on Communications, vol. 52, pp. 484-489, March 2004.

[6] M. M. K. Howlader and B. D. Woerner, "Decoder-assisted frame synchronization for packet transmission," IEEE J. Sel. Areas Comm., vol. 19, pp. 2231-2345, December 2001.

[7] H. Huh and J. V. Krogmeier, "A unified approach to optimum frame synchronization," IEEE Trans. Wireless Comm., vol. 5, pp. 3700-3711, December 2006

[8] S. Houcke and G. Sicot, "Blind frame synchronization for block code," in Proc. of EUSIPCO, European Sig. Proc., September 2006.

[9] R. Imad, S. Houcke, and C. Douillard, "Blind frame synchronization on gaussian channel," in Proc. of EUSIPCO, European Sig. Proc., September 2007.

[10] S. Houcke, G. Sicot, and M. Debbah, "Blind detection for block interleaved division multiple access," in Proc. of GLOBECOM, 2006.

[11] J. Sun, "Synchronization for capacity-approaching coded communication systems," Ph.D. dissertation, College of Engineering and Mineral Resources at West Virginia University, 2004.

[12] J. Sun and M. C. Valenti, "Optimum frame synchronization for preamble-less packet transmission of turbo codes," Asilomar Conference on Signals, Systems, and Computers, vol. 1, pp. 1126-1130, November 2004.

[13] J. Hagenauer, E. Offer, and L. Papke, "Iterative decoding of binary block and convolutional codes," IEEE trans. on Information Theory, vol. 42, no. 2, pp. 429-445, March 1996.

[14] J. Chen and M. P. Fossorier, "Near optimum universal belief propagation based decoding of low-density parity check codes," IEEE Transactions on Communications, vol. 50, pp. 406-414, March 2002. 